This item was submitted to Loughborough's Research Repository by the author.

Items in Figshare are protected by copyright, with all rights reserved, unless otherwise indicated.

\title{
Outage analysis and power allocation in uplink non-orthogonal multiple access systems
}

\section{PLEASE CITE THE PUBLISHED VERSION}

https://doi.org/10.1109/LCOMM.2017.2769088

\section{PUBLISHER}

Institute of Electrical and Electronics Engineers

\section{VERSION}

NA (Not Applicable or Unknown)

\section{PUBLISHER STATEMENT}

This work is made available according to the conditions of the Creative Commons Attribution 3.0 Unported (CC BY 3.0) licence. Full details of this licence are available at: http://creativecommons.org/licenses/by/3.0/

\section{LICENCE}

CC BY 3.0

\section{REPOSITORY RECORD}

Liu, Ye, Mahsa Derakhshani, and Sangarapillai Lambotharan. 2019. "Outage Analysis and Power Allocation in Uplink Non-orthogonal Multiple Access Systems”. figshare. https://hdl.handle.net/2134/27373. 


\title{
Outage Analysis and Power Allocation in Uplink Non-Orthogonal Multiple Access Systems
}

\author{
$\mathrm{Ye} \mathrm{Liu}^{\circledR}$, Member, IEEE, Mahsa Derakhshani ${ }^{\circledR}$, Member, IEEE, \\ and Sangarapillai Lambotharan, Senior Member, IEEE
}

\begin{abstract}
We propose a tractable expression for the outage probability in single-cell uplink non-orthogonal multiple access (NOMA) systems serving an arbitrary number of users. The expression is obtained by approximating the interuser interference using a shifted-gamma distributed random variable. We then formulate and propose an efficient iterative algorithm for the outage-constrained min-max power allocation problem for the NOMA system. To give a rigorous comparison, we solve the outage-constrained min-max power allocation problem for the orthogonal multiple access (OMA) counterpart where both the user power allocation and the radio resource division pattern are optimized. Simulations confirm the accuracy of the derived outage probability expression for the NOMA system. Also, we demonstrate that fairness among users in terms of transmission power can be achieved by NOMA. Moreover, NOMA can bring significant power savings to the users as compared with OMA.
\end{abstract}

Index Terms-Non-orthogonal multiple access, outage probability, fairness, power allocation.

\section{INTRODUCTION}

B Y LEVERAGING the successive interference cancellation (SIC) capabilities at the receivers, non-orthogonal multiple access (NOMA) is regarded as a promising technique to improve spectral and power efficiency for the next generation of cellular networks [1]. Extensive efforts have been devoted to exploring the potential gains of applying NOMA, assuming that perfect channel state information (CSI) is available [2]-[5].

In single-cell downlink systems with only statistical knowledge of CSI available at the transmitter, [6] shows that for a fixed target data rate, NOMA achieves higher diversity order compared to orthogonal multiple access (OMA) schemes in terms of the outage probability. Outage-constrained power allocation optimization was then studied in [7]. Outage analysis of single-cell uplink NOMA systems was studied in [8]-[11], but the analyses are limited to the case of at most three users.

Our work considers a more generalized scenario where an arbitrary number of users are served by a single-cell NOMA system. The challenge of such an outage analysis is to model the randomness of the interference power from an arbitrary number of users. We resolve this difficulty by approximating

Manuscript received September 27, 2017; accepted October 26, 2017. Date of publication November 2, 2017; date of current version February 9, 2018. This work was supported by the Engineering and Physical Science Research Council of the U.K., EPSRC, under Grant EP/M015475. The associate editor coordinating the review of this paper and approving it for publication was F. Wang. (Corresponding author: Ye Liu.)

The authors are with the Signal Processing and Networks Research Group, Wolfson School, Loughborough University, Leicestershire LE11 3TU, U.K. (e-mail: y.liu6@lboro.ac.uk; m.derakhshani@lboro.ac.uk; s.lambotharan@lboro.ac.uk).

Digital Object Identifier 10.1109/LCOMM.2017.2769088 the interference power as a shifted-gamma distributed random variable (RV) [12], which leads to an accurate and tractable closed-form expression for the outage probability. Simulations confirm the accuracy of the derived outage expression.

Based on the developed outage expression of the NOMA system, we formulate the outage-constrained min-max uplink power allocation problem to achieve fairness on the transmission power among the users. Such a fairness consideration is important for prolonging the lifetime of a battery-powered network [13] that is expected to be proliferated in the coming $5 \mathrm{G}$ era and has, to the best of our knowledge, not been studied. The power allocation problem is formulated as a signomial geometric program (SGP), and an efficient iterative algorithm that converges to an optimal solution is proposed.

For a rigorous comparison, we formulate the same power control problem for the OMA system, where the transmission power and the radio resource division pattern (either in time or frequency) are jointly optimized. We show that the bisection method can solve the OMA problem optimally. Simulation studies demonstrate that NOMA can significantly reduce the transmission power of the users while achieving the same level of fairness as OMA in terms of transmission power.

\section{SySTEM MOdEL}

Consider a single-cell uplink system where a single-antenna base station (BS) serves $K$ single-antenna users. Let $\mathcal{K}$ be the set of the user indices. The channel gain between user $k$ and the BS is modeled as $l_{k} \cdot\left|h_{k}\right|^{2}$, where $l_{k}$ gives the path loss and $h_{k}$ denotes the small-scale fading channel coefficient. We assume that the users are quasi-static. Also, without loss of generality, assume $l_{1} \geq l_{2} \geq \cdots \geq l_{K}$. We use Rayleigh fading to model the small-scale fading between the BS and the users, such that $\left|h_{k}\right|^{2}$ is exponentially distributed with rate parameter one. Moreover, we assume that $\left|h_{j}\right|^{2}$ and $\left|h_{k}\right|^{2}$ are independent for all pairs of $j$ and $k$, where $j \neq k$ and $j, k \in \mathcal{K}$.

In the NOMA system, each user occupies the single available resource block (RB) with a bandwidth of one $\mathrm{Hz}$ and time duration of one second. SIC is performed at the BS with decoding order $1,2, \ldots, K$, such that the BS treats the signals from users $k+1, k+2, \ldots, K$ as interference when decoding the signal of user $k$. Denote $\rho_{k}$ as the transmission signal-tonoise ratio (SNR) of user $k$. With perfect SIC, the achievable rate of user $k$ in the NOMA system is

$$
R_{k}^{\mathrm{NOMA}} \triangleq \begin{cases}\ln \left(1+\frac{\rho_{k} l_{k}\left|h_{k}\right|^{2}}{\sum_{j=k+1}^{K} \rho_{j} l_{j}\left|h_{j}\right|^{2}+1}\right), & k \leq K-1, \\ \ln \left(1+\rho_{k} l_{k}\left|h_{k}\right|^{2}\right), & k=K .\end{cases}
$$

For the OMA system, assume the single $\mathrm{RB}$ is divided (either in time or frequency) into $K$ parts, where 
$t_{k} \in[0,1]$ is the size of the $k$-th part and user $k$ occupies the $k$ th part exclusively. Then, user $k$ can achieve the following rate

$$
R_{k}^{\mathrm{OMA}}=t_{k} \cdot \ln \left(1+\rho_{k} l_{k}\left|h_{k}\right|^{2} \cdot t_{k}^{-1}\right) .
$$

We study the min-max power allocation problem for both NOMA and OMA systems under outage constraints, i.e.,

$\operatorname{minimize} \max \left\{\rho_{k}, k \in \mathcal{K}\right\}, \quad$ subject to $\mathrm{P}_{\text {out }, k} \leq \epsilon_{k}, \forall k$,

where $\epsilon_{k}$ gives the tolerable outage probability of user $k$. The formulation in (3) aims to achieve user fairness in terms of transmission power.

\section{NOMA OUTAGE ANAlysis ANd Power Allocation}

We derive a tractable outage probability expression for the uplink NOMA system. Then, we propose an iterative algorithm to solve the power allocation problem in (3).

\section{A. Outage Probability in Uplink NOMA Systems}

In the SIC decoding process, $R_{k}^{\mathrm{NOMA}}$ gives the achievable rate of user $k$, given that the BS decodes the signals from users $1,2, \ldots, k-1$ successfully. Therefore, the outage probability of user $k$ in the NOMA system can be defined as [6], [8],

$$
\mathrm{P}_{\text {out }, k}^{\mathrm{NOMA}} \triangleq 1-\mathrm{P}\left\{D_{1}^{\text {NOMA }} \cap D_{2}^{\text {NOMA }} \cap \cdots \cap D_{k}^{\text {NOMA }}\right\},
$$

where $\mathrm{P}\{\cdot\}$ gives the probability of an event, $D_{k}^{\mathrm{NOMA}}$ is the event of $R_{k}^{\mathrm{NOMA}} \geq \hat{R}_{k}$, and $\hat{R}_{k}$ is the target rate of user $k$. Define $\varphi_{k} \triangleq e^{\hat{R}_{k}}-1 \forall k<K$. When $l_{k} \gg \varphi_{k} l_{k+1} \forall k<K$ holds, Appendix shows that

$$
\mathrm{P}_{\mathrm{out}, k}^{\mathrm{NOMA}} \approx 1-\prod_{j=1}^{k} \mathrm{P}\left\{D_{j}^{\mathrm{NOMA}}\right\} .
$$

Define $g_{k} \triangleq 2 \cdot\left|h_{k}\right|^{2}$ such that $g_{k}$ follows the chi-squared distribution with two degrees of freedom. We have

$$
\mathrm{P}\left\{D_{k}^{\mathrm{NOMA}}\right\}=\mathrm{P}\left\{R_{k}^{\mathrm{NOMA}} \geq \hat{R}_{k}\right\}=\mathrm{P}\left\{\rho_{k} l_{k} g_{k}-y_{k} \geq 2 \varphi_{k}\right\},
$$

where

$$
y_{k} \triangleq \begin{cases}\varphi_{k} \sum_{j=k+1}^{K} \rho_{j} l_{j} g_{j}, & k \leq K-1, \\ 0, & k=K\end{cases}
$$

gives the scaled interference experienced by user $k$. Note that $y_{k}$ is the weighted sum of chi-squared RVs and can be accurately approximated by a shifted-gamma RV as [12]

$$
y_{k} \approx \tilde{y}_{k} \triangleq \alpha_{k} q_{k}+2 \beta_{k},
$$

where $q_{k}$ is a gamma distributed RV with shape parameter $d_{k}$ and scale parameter two, $\alpha_{k}, \beta_{k}$, and $d_{k}$ are given as

$$
\begin{aligned}
& \alpha_{k}=\varphi_{k} \cdot \sum_{j=k+1}^{K} \rho_{k}^{3} l_{k}^{3} \cdot\left[\sum_{j=k+1}^{K} \rho_{j}^{2} l_{j}^{2}\right]^{-1}, \quad k \leq K-1, \\
& \beta_{k}=\varphi_{k} \cdot\left[\sum_{j=k+1}^{K} \rho_{j} l_{j}-\frac{\left(\sum_{j=k+1}^{K} \rho_{j}^{2} l_{j}^{2}\right)^{2}}{\sum_{j=k+1}^{K} \rho_{j}^{3} l_{j}^{3}}\right], \quad k \leq K-1, \\
& d_{k}=\left(\sum_{j=k+1}^{K} \rho_{j}^{2} l_{j}^{2}\right)^{3} \cdot\left(\sum_{j=k+1}^{K} \rho_{j}^{3} l_{j}^{3}\right)^{-2}, \quad k \leq K-1,
\end{aligned}
$$

and $\alpha_{K}=\beta_{K}=d_{K}=0$ since $y_{K}=0$. Note that $\beta_{K-1}=0$ because from (7), $y_{K-1}$ is a weighted chi-squared distributed RV with no shift. Moreover, simple manipulations show that $\beta_{k} \geq 0$ for $k \leq K-2$. Then, from (6) and (9), we approximate $\mathrm{P}_{\mathrm{dec}, k}^{\mathrm{NOMA}}$ as

$$
\mathrm{P}\left\{D_{k}^{\mathrm{NOMA}}\right\} \approx \mathrm{P}\left\{\rho_{k} l_{k} g_{k}-\tilde{y}_{k} \geq 2 \varphi_{k}\right\} .
$$

To calculate (13), first notice that $g_{k}$ and $\tilde{y}_{k}$ are independent. The probability density functions (PDFs) of $g_{k}$ and $\tilde{y}_{k}$ are

$$
f_{g_{k}}=\frac{1}{2} e^{-\frac{g_{k}}{2}}, \quad f_{\tilde{y}_{k}}=\frac{\left(\tilde{y}_{k}-2 \beta_{k}\right)^{d_{k}-1} \cdot e^{\left(-\frac{y_{k}-2 \beta_{k}}{2 \alpha_{k}}\right)}}{\Gamma\left(d_{k}\right) \cdot\left(2 \alpha_{k}\right)^{d_{k}}},
$$

where $\Gamma(\cdot)$ is the gamma function. Notice that $\tilde{y}_{k} \geq 2 \beta_{k}$ since $\tilde{y}_{k}$ is a gamma-distributed RV shifted by $2 \beta_{k}$. Then,

$$
\begin{aligned}
\mathrm{P}\left\{\rho_{k} l_{k} g_{k}-\tilde{y}_{k}<2 \varphi_{k}\right\} & \\
= & \int_{2 \beta_{k}}^{+\infty} \int_{0}^{\frac{\tilde{y}_{k}+2 \varphi_{k}}{\rho_{k} l_{k}}} f_{g_{k}} f_{\tilde{y}_{k}} d g_{k} d \tilde{y}_{k} \\
& =1-\left(\rho_{k} l_{k}\right)^{d_{k}}\left(\rho_{k} l_{k}+\alpha_{k}\right)^{-d_{k}} e^{-\frac{\varphi_{k}+\beta_{k}}{\rho_{k}}} .
\end{aligned}
$$

Based on (13) and (15), $\mathrm{P}\left\{D_{k}^{\mathrm{NOMA}}\right\}$ can be approximated as

$$
\mathrm{P}\left\{D_{k}^{\mathrm{NOMA}}\right\} \approx\left(\rho_{k} l_{k}\right)^{d_{k}}\left(\rho_{k} l_{k}+\alpha_{k}\right)^{-d_{k}} e^{-\frac{\varphi_{k}+\beta_{k}}{\rho_{k}}} .
$$

Finally, based on (5) and (16), we obtain a closed-form expression for the outage probability of NOMA user $k$ as

$$
\mathrm{P}_{\mathrm{out}, k}^{\mathrm{NOMA}} \approx 1-\prod_{j=1}^{k}\left(\rho_{j} l_{j}\right)^{d_{j}}\left(\rho_{j} l_{j}+\alpha_{j}\right)^{-d_{j}} e^{-\frac{\varphi_{j}+\beta_{j}}{\rho_{j} l_{j}}} .
$$

\section{B. Min-Max Power Allocation for Uplink NOMA Systems}

Based on (17), we rewrite (3) for the NOMA system as

$\underset{\rho, z, \alpha, \beta, d}{\operatorname{minimize}} z, \quad$ subject to (10), (11), (12), $z \geq \rho_{k} \forall k, \quad$ (18a)

$$
\prod_{j=1}^{k}\left(\rho_{j} l_{j}\right)^{d_{j}}\left(\rho_{j} l_{j}+\alpha_{j}\right)^{-d_{j}} e^{-\frac{\varphi_{j}+\beta_{j}}{\rho_{j} l_{j}}} \geq \hat{\epsilon}_{k} \quad \forall k,
$$

where $z$ denotes the maximum transmission SNR among all users, $\hat{\epsilon}_{k} \triangleq 1-\epsilon_{k} \forall k$, (18b) means $\mathrm{P}_{\text {out }, k}^{\mathrm{NOMA}} \leq \epsilon_{k} \forall k$, $\boldsymbol{\rho} \triangleq\left[\rho_{1}, \rho_{2}, \ldots, \rho_{K}\right], \boldsymbol{\alpha} \triangleq\left[\alpha_{1}, \alpha_{2}, \ldots, \alpha_{K-1}, 0\right], \boldsymbol{\beta} \triangleq$ $\left[\beta_{1}, \beta_{2}, \ldots, \beta_{K-2}, 0,0\right]$, and $\boldsymbol{d} \triangleq\left[d_{1}, d_{2}, \ldots, d_{K-1}, 0\right]$.

To solve the problem in (18), we transform it to an SGP whose optimal solution can be efficiently found by solving a series of geometric programs (GPs) [14], where GPs can be transformed into convex problems and solved by off-the-shelf optimization toolboxes. The definition of an SGP can be found in [14] and is omitted here for simplicity.

We now present the two steps needed to transform (18) into an SGP. By some manipulations, (18b) can be rewritten as

$$
\sum_{j=1}^{k}\left[\frac{\varphi_{j} l_{j}^{-1} \rho_{j}^{-1}}{\ln \left(\hat{\epsilon}_{k}^{-1}\right)}+\frac{\beta_{j} l_{j}^{-1} \rho_{j}^{-1}}{\ln \left(\hat{\epsilon}_{k}^{-1}\right)}+\frac{d_{j} \lambda_{k}}{\ln \left(\hat{\epsilon}_{k}^{-1}\right)}\right] \leq 1,
$$

where we introduce new variables $\left\{\lambda_{k} \mid k \in \mathcal{K}\right\}$ that give rise to the following new constraints

$$
e^{\lambda_{k}}=1+\alpha_{k} \rho_{k}^{-1} l_{k}^{-1}, \quad \forall k .
$$

The Taylor series of the exponential function suggests that

$$
e^{\lambda_{k}}=\sum_{i=0}^{\infty} \frac{\lambda_{k}^{i}}{i !}=1+\sum_{i=1}^{\infty} \frac{\lambda_{k}^{i}}{i !} \approx 1+\sum_{i=1}^{U} \frac{\lambda_{k}^{i}}{i !},
$$


where $U$ is a sufficiently large positive integer such that the approximation in (21) is accurate. Then, (20) is replaced by

$$
f_{1, k} \triangleq \sum_{i=1}^{U}(i !)^{-1} \alpha_{k}^{-1} \rho_{k} l_{k} \lambda_{k}^{i}=1, \quad \forall k .
$$

Let $v_{1, k} \triangleq \sum_{j=k+1}^{K} \rho_{j}^{2} l_{j}^{2}$ and $v_{2, k} \triangleq \sum_{j=k+1}^{K} \rho_{j}^{3} l_{j}^{3}$ for $k \leq$ $K-1$. In the second step of the SGP formulation, we transform the constraints in (10), (11), and (12) into (23).

$$
\begin{aligned}
& f_{2, k} \triangleq \varphi_{k} \cdot \alpha_{k}^{-1} \cdot v_{2, k} \cdot v_{1, k}^{-1}=1, \quad k \leq K-1, \\
& f_{3, k} \triangleq\left(\frac{\beta_{k}}{\varphi_{k}}+\frac{v_{1, k}^{2}}{v_{2, k}}\right) \cdot\left(\sum_{j=k+1}^{K} \rho_{j} l_{j}\right)^{-1}=1, \quad k \leq K-1,
\end{aligned}
$$

$f_{4, k} \triangleq d_{k} \cdot v_{1, k}^{-3} \cdot v_{2, k}^{2}=1, \quad k \leq K-1$,

$f_{5, k} \triangleq v_{1, k}^{-1} \cdot \sum_{j=k+1}^{K} \rho_{j}^{2} l_{j}^{2}=1, \quad k \leq K-1$,

$f_{6, k} \triangleq v_{2, k}^{-1} \cdot \sum_{j=k+1}^{K} \rho_{j}^{3} l_{j}^{3}=1, \quad k \leq K-1$.

The transformation of (18) to an SGP is complete if we replace (10), (11), (12), and (18b) by (19), (22), and (23).

The successive convex approximation (SCA) algorithm proposed in [14] can be used to obtain a Karush-KuhnTucker (KKT) point of an SGP. To apply the SCA, first realize that the SGP formulation of (18) is equivalent to

$$
\begin{aligned}
& \underset{\boldsymbol{\rho}, z, \boldsymbol{\alpha}, \boldsymbol{\beta}, \boldsymbol{\lambda}, \boldsymbol{v}, \boldsymbol{d}, \boldsymbol{s}}{\operatorname{minimize}} z+\sum_{j=1}^{6} \sum_{k} w_{j, k} s_{j, k}, \\
& \text { subject to } z \geq \rho_{k}, \quad f_{j, k} \leq 1, s_{j, k}^{-1} f_{j, k}^{-1} \leq 1, \\
& s_{j, k} \geq 1, \quad \forall j, k
\end{aligned}
$$

when all the auxiliary variables $s_{j, k}$ are equal to ones, where $w_{j, k}$ is a positive weight that forces $s_{j, k}$ to take small values and $\mathbf{s} \triangleq\left\{s_{j, k} \mid j=1,2, \ldots, 6, k \in \mathcal{K}\right\}$. Then, the arithmetic-geometric mean approximation (AGMA) is applied to $s_{j, k}^{-1} f_{j, k}^{-1} \forall j, k$, such that a GP approximation of (24) is obtained [14]. As an iterative algorithm, the SCA starts by choosing an initial point to perform the AGMA, which formulates the first GP. The solution to the first GP is used to perform the AGMA in order to formulate the second GP, and such process is repeated until some stopping criterion is met. The SCA converges to a KKT point of an SGP when $s_{j, k}=1 \forall j, k$, where the proof can be found in [14]. In the simulations, for practical reasons, we stop the SCA when $\left\{\rho_{k} \mid k \in \mathcal{K}\right\}$ satisfies the outage targets and $s_{j, k}<1.01, \forall j, k$.

\section{OMA Min-MaX POWER Allocation}

We present two OMA schemes. The first OMA scheme jointly optimizes the power allocation and the RB partition for each user. The second OMA scheme optimizes the power allocation where each user occupies one part of the RB of the same size, i.e., $t_{k}=K^{-1} \forall k$. The two OMA schemes represent two extremes in terms of the complexity of RB partitioning.

\section{A. Joint Power and Radio Resource Optimization}

Define $\phi_{k}\left(t_{k}, \rho_{k}\right) \triangleq t_{k} \rho_{k}^{-1} l_{k}^{-1}\left(e^{\hat{R}_{k} / t_{k}}-1\right)$, where $k \in \mathcal{K}$. The outage probability of user $k$ in the OMA system is

$$
\mathrm{P}_{\mathrm{out}, k}^{\mathrm{OMA}} \triangleq \mathrm{P}\left\{R_{k}^{\mathrm{OMA}}<\hat{R}_{k}\right\}=1-e^{-\phi_{k}\left(t_{k}, \rho_{k}\right)} .
$$

Then, we rewrite the problem in (3) for the OMA system as

$$
\begin{aligned}
& \underset{\rho, \mathbf{t}, z}{\operatorname{minimize}} z, \quad \text { subject to } z \geq \rho_{k} \forall k, \\
& \sum_{k=1}^{K} t_{k}=1, \quad \mathrm{P}_{\mathrm{out}, k}^{\mathrm{OMA}}=1-e^{-\phi_{k}\left(t_{k}, \rho_{k}\right)} \leq \epsilon_{k} \forall k,
\end{aligned}
$$

where $\mathbf{t} \triangleq\left[t_{1}, t_{2}, \ldots, t_{K}\right]$. Denote $\mathbf{a}^{*}$ as the optimal value of a variable vector a with an arbitrary length. The next lemma shows the conditions that the optimal solution to (26) satisfies.

Lemma 1: The unique global optimal solution to (26) satisfies $1-e^{-\phi_{k}\left(t_{k}^{*}, \rho_{k}^{*}\right)}=\epsilon_{k}$ and $z^{*}=\rho_{k}^{*}$ for all $k \in \mathcal{K}$.

Proof: One can show that $\mathrm{P}_{\text {out }, k}^{\mathrm{OMA}}$ is a decreasing function of $\rho_{k}$ and $t_{k}$. For a fixed $t_{k}, \rho_{k}$ is minimized when $\mathrm{P}_{\text {out }, k}^{\mathrm{OMA}}=\epsilon_{k}$; further increasing $\rho_{k}$ is suboptimal since it may increase $z$.

Then, suppose $\left\{\boldsymbol{\rho}^{\prime}, \mathbf{t}^{\prime}, z^{\prime}\right\}$ is a solution of (26), where $\rho_{j}^{\prime} \neq \rho_{k}^{\prime}$ and assume without loss of generality that $\rho_{j}^{\prime}>\rho_{k}^{\prime}$. We can decrease $\rho_{j}^{\prime}$ by $\delta_{\rho_{j}}$ and increase $t_{j}^{\prime}$ such that $\mathrm{P}_{\text {out }, j}^{\mathrm{OMA}}=$ $\epsilon_{j}$ is maintained, where $\delta_{\rho_{j}}>0$. Similarly, we can increase $\rho_{k}^{\prime}$ by $\delta_{\rho_{k}}$ and decrease $t_{k}^{\prime}$ such that $\mathrm{P}_{\text {out }, k}^{\mathrm{OMA}}=\epsilon_{k}$, where $\delta_{\rho_{k}}>0$. We can adjust until $\rho_{j}^{\prime}-\delta_{\rho_{j}}=\rho_{k}^{\prime}+\delta_{\rho_{k}}$. Also, the adjustment will improve $z^{\prime}$ by $\delta_{\rho_{j}}$ if $\rho_{j}^{\prime}$ is the largest element in $\rho^{\prime}$, which justifies that $z^{*}=\rho_{1}^{*}=\rho_{2}^{*}=\cdots=\rho_{K}^{*}$. The uniqueness of $z^{*}$ is due to the linearity of the objective function.

Lemma 1 implies the following at the global optimum, i.e.,

$$
z^{*} \cdot l_{k} \cdot \ln \left(\hat{\epsilon}_{k}^{-1}\right)=t_{k}^{*} \cdot\left(e^{\hat{R}_{k} / t_{k}^{*}}-1\right), \quad \forall k,
$$

where some calculus shows that $t_{k} \cdot\left(e^{\hat{R}_{k} / t_{k}}-1\right)$ is a decreasing function of $t_{k}$. Also, $t_{k}^{*}$ is uniquely determined by $z^{*}$ and other parameters in (27). This implies that $\sum_{k=1}^{K} t_{k}>1$ if $z<z^{*}$, and $\sum_{k=1}^{K} t_{k}<1$ if $z>z^{*}$. Then, $z^{*}$ and $\left\{t_{k}^{*} \mid k \in \mathcal{K}\right\}$ can be found by a nested bisection procedure, where the outerbisection searches for $z$ and the inner-bisection searches for $\left\{t_{k} \mid k \in \mathcal{K}\right\}$ that satisfies (27) for a given $z$.

\section{B. Power Allocation When $t_{k}=K^{-1} \forall k$}

When $t_{k}=K^{-1}, \rho_{k}$ must satisfy $1-e^{-\phi_{k}\left(K^{-1}, \rho_{k}\right)} \leq \epsilon_{k}$, where $1-e^{-\phi_{k}\left(K^{-1}, \rho_{k}\right)}$ is a decreasing function of $\rho_{k}$. Because the objective is to minimize the maximum of $\rho_{k}$ among all $k$, the optimal solution of $\rho_{k}$ satisfies $1-$ $e^{-\phi_{k}\left(K^{-1}, \rho_{k}\right)}=\epsilon_{k}$. Therefore, the optimal solution of (26) when $t_{k}=K^{-1}$ is $z^{*}=\max _{k}\left\{\rho_{k}^{*}\right\}$, where $\rho_{k}^{*}=K^{-1} l_{k}^{-1}$ $\left(1-e^{\hat{R}_{k} K}\right)\left[\ln \left(\hat{\epsilon}_{k}\right)\right]^{-1} \forall k$.

\section{Simulation Studies}

For all simulations, we set $\epsilon_{k}=0.1 \forall k \in \mathcal{K}$. The distance between the BS and the $k$-th user is given as $\zeta_{k} \triangleq$ $10^{k-1} \forall k \in \mathcal{K}$. The path loss exponent is set to three such that $l_{k}=\zeta_{k}^{-3} \forall k \in \mathcal{K}$.

Fig. 1 shows the accuracy of the approximation in (17), where $K=5, \hat{R}_{k}=1 \mathrm{nat} / \mathrm{sec} / \mathrm{Hz} \forall k$, and $\rho_{1}=\rho_{2}=\cdots=$ $\rho_{K}$. We obtain the empirical results by counting the outage events as defined in (4) in $10^{6}$ random channel realizations. The maximum difference between the empirical results and the analytical results is $1.5 \times 10^{-3}$, verifying the accuracy of (17).

Fig. 2 compares the performance of OMA and NOMA. In the legends, "OMA eq." represents the OMA system where $t_{k}=K^{-1} \forall k \in \mathcal{K}$, and "OMA opt." accounts for the OMA 


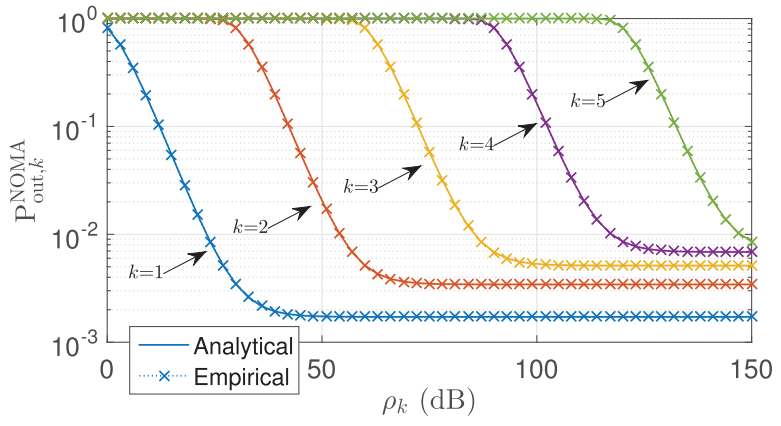

Fig. 1. Verification of the accuracy on the analytical expression of $\mathrm{P}_{\text {out }, k}^{\mathrm{NOMA}}$ in (17), where $K=5$ and $\rho_{1}=\rho_{2}=\cdots=\rho_{K} \cdot k$ is the user index.

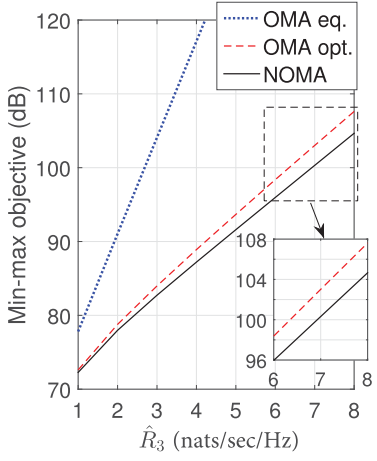

(a)

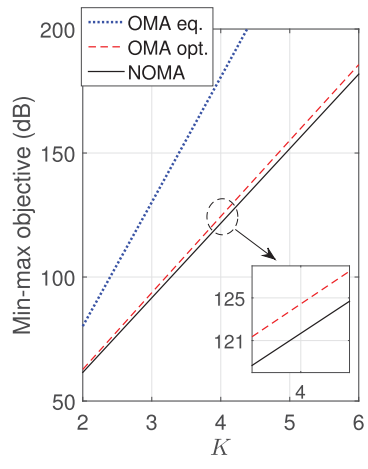

(b)
Fig. 2. Comparison between the NOMA and the OMA schemes after the min-max power allocation optimizations.

system where both $t_{k}$ and $\rho_{k}$ are optimized for all $k \in \mathcal{K}$. The results in Fig. 2 provide a more holistic view on the benefit of NOMA against OMA as compared to other studies [7], [8] which do not consider a fully optimized OMA system.

More specifically, Fig. 2(a) studies the scenario where $K=3, \hat{R}_{1}=\hat{R}_{2}=1 \mathrm{nat} / \mathrm{sec} / \mathrm{Hz}$, and $\hat{R}_{3}$ increases from one to eight nats/sec/Hz. Observe that NOMA reduces the maximum transmission power among the users by about $3 \mathrm{~dB}$ compared to OMA opt. when $\hat{R}_{3}=8 \mathrm{nat} / \mathrm{sec} / \mathrm{Hz}$, and NOMA can reduce the same quantity tremendously when compared to OMA eq.. For the fairness among $\rho_{k} \forall k \in \mathcal{K}$, we evaluate the Jain's index $\frac{\left(\sum_{k=1}^{K} \rho_{k}\right)^{2}}{K \cdot \sum_{k=1}^{K} \rho_{k}^{2}} \in\left[K^{-1}, 1\right]$, where a Jain's index of 1 means absolute fairness, i.e., $\rho_{1}=\rho_{2}=\cdots=\rho_{K}$. We find out that the Jain's indices for NOMA, OMA opt., and OMA eq. are respectively 1,1 , and around 0.33 , which suggests that the NOMA system can provide the same level of fairness as compared to the fully optimized OMA counterpart. Using MOSEK as the GP solver, the SCA efficiently solves (18) in 5.4 seconds on average for each point in Fig. 2(a) on a desktop without parallel computing.

Fig. 2(b) compares NOMA against the OMA schemes when $K$ varies, where $\hat{R}_{K}=5$ nats $/ \mathrm{sec} / \mathrm{Hz}$ and $\hat{R}_{k}=1$ nats $/ \mathrm{sec} / \mathrm{Hz}$ for $k<K$. Observe that NOMA outperforms OMA opt. by about $3 \mathrm{~dB}$ when $K=4$, and the gap between NOMA and the OMA opt. increases as $K$ increases. Also, NOMA outperforms OMA eq. tremendously.

\section{CONCLUSION}

An accurate and tractable approximation on the outage probabilities in a single-cell uplink NOMA system with an arbitrary number of users was obtained. Subsequently, the outage-constrained min-max power allocation problems for both NOMA and OMA systems were solved, where the power allocation and the radio resource partition in the OMA system were jointly optimized for a rigorous comparison against the NOMA system. The OMA problem could be solved optimally using the bisection method. An iterative algorithm for the NOMA power allocation problem was proposed that converges to a KKT point. Simulations demonstrated that NOMA could achieve the same level of fairness as OMA in terms of uplink transmission power. Also, results showed significant power saving gained by applying NOMA and the efficiency of the iterative algorithm.

\section{APPENDIX: INDEPENDENCE ASSUMPTION IN (5)}

The events $D_{1}^{\text {NOMA }}$ and $D_{2}^{\text {NOMA }}$ are respectively $\varphi_{1}^{-1} \rho_{1}$ $l_{1}\left|h_{1}\right|^{2}-\rho_{2} l_{2}\left|h_{2}\right|^{2}>\sum_{j=3}^{K} \rho_{j} l_{j}\left|h_{j}\right|^{2}+1$ and $\varphi_{2}^{-1} \rho_{2} l_{2}\left|h_{2}\right|^{2}>\sum_{j=3}^{K} \rho_{j} l_{j}\left|h_{j}\right|^{2}+1$. Because $\varphi_{1}^{-1} l_{1} \gg l_{2}$, $\rho_{2} \quad l_{2}\left|h_{2}\right|^{2}$ has little effect on $\mathrm{P}\left\{D_{1}^{\mathrm{NOMA}}\right\}$, such that $\mathrm{P}\left\{D_{1}^{\mathrm{NOMA}}\right\} \approx \mathrm{P}\left\{\varphi_{1}^{-1} \rho_{1} l_{1}\left|h_{1}\right|^{2}>\sum_{j=3}^{K} \rho_{j} l_{j}\left|h_{j}\right|^{2}+1\right\}$. Then, $D_{1}^{\text {NOMA }}$ and $D_{2}^{\text {NOMA }}$ are independent by realizing that $h_{1}$ and $h_{2}$ are independent. The same argument can be applied to argue that $\mathrm{P}\left\{D_{j}^{\mathrm{NOMA}} \mid D_{k}^{\mathrm{NOMA}}\right\} \approx \mathrm{P}\left\{D_{j}^{\mathrm{NOMA}}\right\}$ for any $j<k$ where $k \geq 2$, and the assumption in (5) follows.

\section{REFERENCES}

[1] S. M. R. Islam, M. Zeng, and O. A. Dobre, "NOMA in 5G systems: Exciting possibilities for enhancing spectral efficiency," IEEE 5G Tech. Focus, vol. 1, no. 2, May 2017.

[2] M. Mollanoori and M. Ghaderi, "Uplink scheduling in wireless networks with successive interference cancellation," IEEE Trans. Mobile Comput., vol. 13, no. 5, pp. 1132-1144, May 2014.

[3] L. Lei et al., "Power and channel allocation for non-orthogonal multiple access in 5G systems: Tractability and computation," IEEE Trans. Wireless Commun., vol. 15, no. 12, pp. 8580-8594, Dec. 2016.

[4] M.-R. Hojeij et al., "Waterfilling-based proportional fairness scheduler for downlink non-orthogonal multiple access," IEEE Wireless Commun. Lett., vol. 6, no. 2, pp. 230-233, Apr. 2017.

[5] Z. Wei et al., "A survey of downlink non-orthogonal multiple access for 5G wireless communication networks," ZTE Commun., vol. 14, no. 4, pp. 17-25, Oct. 2016.

[6] Z. Ding et al., "On the performance of non-orthogonal multiple access in 5G systems with randomly deployed users," IEEE Signal Process. Lett., vol. 21, no. 12, pp. 1501-1505, Dec. 2014.

[7] J. Cui et al., "A novel power allocation scheme under outage constraints in NOMA systems," IEEE Signal Process. Lett., vol. 23, no. 9, pp. 1226-1230, Sep. 2016.

[8] N. Zhang et al., "Uplink nonorthogonal multiple access in 5G systems," IEEE Commun. Lett., vol. 20, no. 3, pp. 458-461, Mar. 2016.

[9] Z. Yang et al., "A general power allocation scheme to guarantee quality of service in downlink and uplink NOMA systems," IEEE Trans. Wireless Commun., vol. 15, no. 11, pp. 7244-7257, Nov. 2016.

[10] Z. Zhang et al., "Downlink and uplink non-orthogonal multiple access in a dense wireless network," IEEE J. Sel. Areas Commun., to be published. [Online]. Available: http://ieeexplore.ieee.org/document/7972929/, doi: 10.1109/JSAC.2017.2724646.

[11] Y. Gao et al., "Theoretical analysis of the dynamic decode ordering SIC receiver for uplink NOMA systems," IEEE Commun. Lett., vol. 21, no. 10, pp. 2246-2249, Oct. 2017.

[12] J.-T. Zhang, "Approximate and asymptotic distributions of chi-squared: Type mixtures with applications," J. Amer. Stat. Assoc., vol. 100, no. 469, pp. 273-285, 2005.

[13] H. Shi et al., "Fairness in wireless networks: Issues, measures and challenges," IEEE Commun. Surveys Tuts., vol. 16, no. 1, pp. 5-24, 1st Quart., 2014.

[14] G. Xu, "Global optimization of signomial geometric programming problems," Eur. J. Oper. Res., vol. 233, no. 3, pp. 500-510, 2014. 